\title{
Self Organizing Classifiers and Niched Fitness
}

\author{
Danilo V. Vargas \\ Graduate School of \\ Information Science and \\ Electrical Engineering, \\ Kyushu University \\ Fukuoka, Japan \\ vargas@cig.ees.kyushu- \\ u.ac.jp
}

\author{
Hirotaka Takano \\ Faculty of Information Science \\ and Electrical Engineering, \\ Kyushu University \\ Fukuoka, Japan \\ takano@cig.ees.kyushu- \\ u.ac.jp
}

\author{
Junichi Murata \\ Faculty of Information Science \\ and Electrical Engineering, \\ Kyushu University \\ Fukuoka, Japan \\ murata@cig.ees.kyushu- \\ u.ac.jp
}

\begin{abstract}
Learning classifier systems are adaptive learning systems which have been widely applied in a multitude of application domains. However, there are still some generalization problems unsolved. The hurdle is that fitness and niching pressures are difficult to balance. Here, a new algorithm called Self Organizing Classifiers is proposed which faces this problem from a different perspective. Instead of balancing the pressures, both pressures are separated and no balance is necessary. In fact, the proposed algorithm possesses a dynamical population structure that self-organizes itself to better project the input space into a map. The niched fitness concept is defined along with its dynamical population structure, both are indispensable for the understanding of the proposed method. Promising results are shown on two continuous multi-step problems. One of which is yet more challenging than previous problems of this class in the literature.
\end{abstract}

\section{Categories and Subject Descriptors}

I.2.11 [Artificial Intelligence]: Distributed Artificial Intelligence - intelligent agents, languages and structures, multiagent systems; I.2.6 [Artificial Intelligence]: Learning

\section{General Terms}

Algorithms, Performance

\section{Keywords}

Self Organization, Self Organizing Systems, Self Organizing Map, Learning Classifier Systems, Reinforcement Learning, Structured Evolutionary Algorithms

\section{INTRODUCTION}

The importance of classifiers to maximize their reward (decrease the error in supervised learning problems or in-

Permission to make digital or hard copies of all or part of this work for personal or classroom use is granted without fee provided that copies are not made or distributed for profit or commercial advantage and that copies bear this notice and the full citation on the first page. To copy otherwise, to republish, to post on servers or to redistribute to lists, requires prior specific permission and/or a fee.

GECCO'13, July 6-10, 2013, Amsterdam, The Netherlands.

Copyright 2013 ACM 978-1-4503-1963-8/13/07 ...\$15.00. crease reward in reinforcement learning problems) with at the same time specializing to their best suited position (niching) were demonstrated by many articles about learning classifier systems (LCS) 29, 22. Throughout the LCS history, there has been an initial focus on strength based systems (e.g., ZCS 33]) which highlight the focus on reward. Later, however, it was realized that much of the niching capability may be lost in strength based systems, because over-general classifiers dominate and high payoff niches become crowded. Accuracy based systems, on the other hand, (e.g., XCS 34 10, XCSF 35]) try a middle ground between niching and reward. By rewarding the accuracy of the reward prediction, accuracy based systems have justifiably better niching than strength based systems (the accuracy-based fitness is more uniform over different niches). For this reason, recent articles have focused more on accuracy-based systems.

However, it is known for some time that XCS systems (and in general, accuracy-based systems) may have problems with generalization 19,8 . Solutions have been proposed for some environments 21], but the hindrance is that different pressures are acting together and balancing them properly for every environment is difficult [13. In other words, the balance between specialized (niched) and generic classifiers is still unsolved.

This article propose to face the dilemma from a different perspective. What if we separate completely the niching problem from the fitness problem? Suppose we could divide the input space into small niches ${ }^{1}$ and evolve the solutions for each niche. By doing this, the fitness for each niche can be strength based because the niches are small and independently defined (not defined by the chromosome and thus not affected by evolution). Moreover, there is not any balance necessary, since niching and fitness pressures were separated from each other.

But then, two questions need to be solved:

1. How can we divide the input space automatically and compactly?

2. How can we compare individuals? Notice that the fitness defined above is relative to the niche!

The answer to the first question lies in using Self Organizing Map (SOM). Self Organizing Map is an unsupervised

\footnotetext{
${ }^{1}$ Concerning the proposed method, the term niche follows the Hutchinsonian niche definition 15. Hutchinsonian niche is an $n$-dimensional hyper-volume composed of environmental features.
} 
artificial neural network 18. It is an algorithm capable of projecting high dimensional spaces into a map. In other words, by using SOM, a multidimensional input space can be divided automatically into the cells of a compact map. In fact, the projection done preserves the topological properties and retains many other relevant information (e.g., data frequency) of the input space.

The second question has a simple answer: they can not be compared. With relative fitness, there is not any easy way of comparing individuals from different niches. Therefore, regarding the fitness selection, the evolutionary algorithm (EA) should be constrained to each niche. Genetic operators, however, are not constrained and may benefit from any population structure present.

Actually, the proposed approach is evaluated at two continuous multi-step problems with promising results. The experiments conducted on two continuous labyrinths demonstrate the capabilities of the proposed approach. One of which is more challenging than the continuous multi-step problems solved by state of the art LCS.

\section{LEARNING CLASSIFIER SYSTEMS IN MULTI-STEP AND/OR CONTINUOUS PROBLEMS}

Learning classifier systems are evolutionary based systems capable of solving problems by the mutual competition and/or cooperation of their solutions. There is a wide and diverse literature. Here we will confine to a brief review of LCS applied to multi-step and/or continuous problems. For a detailed review of the literature, please refer to 29,22 .

LCS with continuous actions were applied to many problems. To begin with, XCSF has been applied to function approximation 35, 9, 28. Other works in function approximation include the LCS with fuzzy logic [30, 6, 11, neuralbased LCS algorithms [5, 6] and genetic programming-based 17. The success of LCS also span the control of robotic arms 25, 7] and navigation problems [4, 14.

However, applications to multi-step problems with continuous actions restrict to the mobile robot in a corridor 4 and the empty room with noise [14]. Complex multi-step problems were solved only for discrete outputs 20 .

\section{STRUCTURED EVOLUTIONARY ALGO- RITHMS}

Structured evolutionary algorithms does not possess a panmictic population. Instead they organize the individuals into a structured population [27, 1].

Two types of structured EAs will be given as examples which are somewhat related to the structure of the proposed method.

The first type is island models (also called distributed genetic algorithms) 3]. Figure 1 shows the structure. Basically, the population is divided into a number of subpopulations ("islands") with few genetic information exchanged between them.

The second type, cellular algorithms are structured evolutionary algorithms where individuals are usually positioned in a vertex of a lattice graph (Figure 2 shows a common cellular structure). They interact solely with adjacent individuals defined by the a neighborhood function 23,2 .

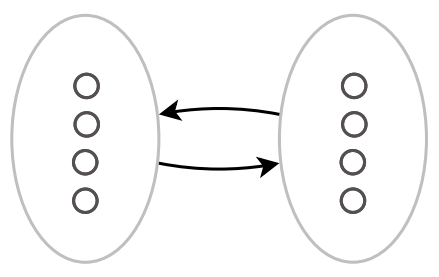

Figure 1: Island model structure. Arrows indicate the infrequent immigration procedure, the circles are the individuals and the oval shapes are the subpopulations.

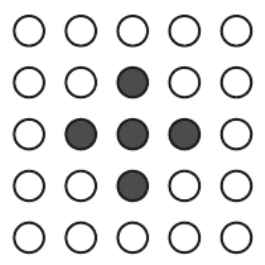

Figure 2: Cellular algorithm structure. Shaded area indicates an example of neighborhood for the central individual.

\section{NEW CONCEPTS}

First, in order to present the proposed method, it is necessary to explain some new concepts and their related literature.

\subsection{Niched Fitness}

A fitness which is relative to a given place or circumstance is called niched fitness. Thus, it does not possess a meaning when compared with another fitness out of this place or circumstance.

This is a new concept. At first glance, it may appear unwise to use niched fitness, because the comparison between individuals from different niches becomes impossible. But there is the benefit of avoiding competition between tasks different in nature. Therefore, every niche existence is protected from other more rewarding and/or more voluminous and/or more frequently accessed niches.

Moreover, niched fitness can be thought as a multiobjectivization procedure 24, because each niched fitness becomes a separate fitness. In fact, niching and multi-objective search are related subjects. The Pareto front created allows the existence of multiple non-dominated individuals (different niches). We consider this a relevant aspect which deserves to be mentioned. The remaining of this article, however, will ignore this aspect and treat the search as a single objective one.

\subsection{SOM Population}

This Section aims to define the SOM population concept. SOM population is a $2 \mathrm{D}$ grid with each cell in the grid having a subpopulation. Moreover, the $2 \mathrm{D}$ grid is a projection map which is not static, i.e., it is part of a SOM which is always self-organizing itself. Figure 3 illustrates the SOM population (SOM's weight vectors are excluded for simplification). Generally speaking, this structure can be seen as a mixture of both island models and cellular algorithms, with 


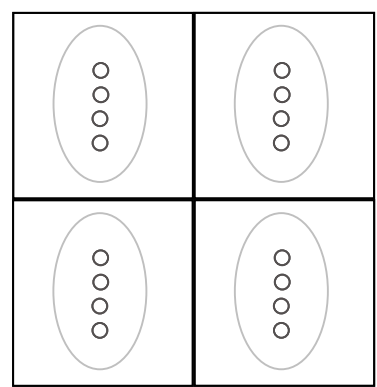

Figure 3: SOM population structure. A self organizing map grid with populations inside each cell.

the additional feature that the structure also changes its properties in relation to the input.

The SOM population's behavior is described below. When an input is given to a SOM population, a competition takes place between the cells of the map. The cell which is closer wins the competition to the input and the SOM cells in the neighborhood are updated (the update make the SOM cell's weight vectors get closer to the input). Then, an individual inside the winner cell acts on the environment and is consequently evaluated by a subsequent reward. The individual chosen to act inside the winner cell is based on a selection procedure which is algorithm specific. (here we use a random selection procedure, see Section 5).

\section{SELF ORGANIZING CLASSIFIERS}

Figure 4 shows a schematic of the overall procedure of Self Organizing Classifiers (SOC). It follows the classic schematic style used in ZCS, XCS and many other papers of the LCS literature 33. Simple classifiers are used. They are made up of action parameters represented by an array of real numbers.

SOC uses a Q-learning based reinforcement scheme with niched fitness. The fitness update of each individual is done using the Widrow-Hoff rule 32 :

$$
F=F+\eta(\hat{F}-F)
$$

where $\eta$ is the learning rate, $F$ is the current fitness and $\hat{F}$ is a new fitness estimate. The fitness estimate of cell cell and classifier $c$ which were activated at time $t-1$ is given by the following equation:

$$
\hat{F}(c, \text { cell })_{t-1}=R_{t-1}+\gamma \max _{c^{\prime} \in \text { cell }}\left\{F\left(c^{\prime}, \text { cell }^{\prime}\right)\right\},
$$

where $R$ is the reward received, $\gamma$ is the discount-factor and $\max _{c \in \text { cell }}\{F(t)\}$ is the maximum fitness of classifier $c^{\prime}$ inside the activated cell cell' at the current cycle $t$.

Similar to learning classifier systems, to decrease computation resources, the structure of the SOM population is implemented as a single array of classifiers with a given numerosity indexed by the SOM population structure. In this manner, the numerosity is defined by the number of indexes a given individual possess.

This article uses a particular SOM population where the subpopulation inside each cell is divided into two groups: one of best individuals and the other of novel individuals. Best and novel individuals have a fixed size of $\beta$ and $\nu$ respec- tively. Considering EA's cycle is an algorithm cycle when the EA is called, the following rules take place:

- Best individuals are the best fitted individuals inside the subpopulation in the last EA's cycle.

- Novel individuals are renewed every EA's cycle (the detailed process is described in the next Section).

The SOM population begins without any classifiers. Classifiers are created when the respective cell wins the competition inside the SOM. In one hand, novel individuals are created as random classifiers. On the other hand, best individuals, when possible, are set equal to another cell's best individuals from the neighborhood ${ }^{2}$ which maximize $\frac{\text { experience }}{\text { chebishevDistance }^{2}}$. If not possible, best individuals are initialized in the same way as the novel individuals.

The system has, as usual in reinforcement learning, cycles of exploration and exploitation. Within the SOM's winning cell in a giving exploration or exploitation cycle a random individual from respectively the novel or best individuals are chosen to act. Moreover, cycles of exploration and exploitation are always alternated (a cycle of exploration is followed by an exploitation cycle and so on).

\subsection{Evolution}

For every cycle that a cell's individual acts, this cell has its experience counter increased. The evolutionary algorithm is called locally on each cell when the cell's experience is greater than $\iota S$. Where $S$ is the number of subpopulation individuals (novel plus best individuals) present on each cell. The parameter $\iota$ defines an experience per individual, above which they should have an accurate fitness evaluation.

By applying the evolutionary algorithm locally, it respects the niched fitness concept. Its procedure consists of sorting the individuals of the given cell according to their fitness. The current best $\beta$ individuals substitute the previous best individuals and the remaining individuals are discarded (the index is removed and the individual numerosity decrease, if the numerosity reaches 0 it is deleted). Novel $\nu$ individuals are created using either:

1. Indexing - A copy from (index to) a randomly selected individual of the entire population;

2. Reproduction - Created by a genetic operator

The two procedures above have equal probabilities.

Motivated by some comparison articles and robustness tests, the differential evolution is chosen as the genetic operator 26, 31, 16. It compares well to even complex optimization algorithms (e.g., Estimation of Distribution Algorithms) 12. The differential evolution's mutant vector is created by randomly choosing three vectors from the SOM's entire population of individuals (individuals with numerosity bigger than one are counted as one).

\section{EXPERIMENTS}

\subsection{Environments}

The experiments were conducted on both Empty Room and One-Wall Maze environments. They are respectively

\footnotetext{
${ }^{2}$ Neighborhood is defined as the cells within a Chebishev distance of less or equal to four
} 


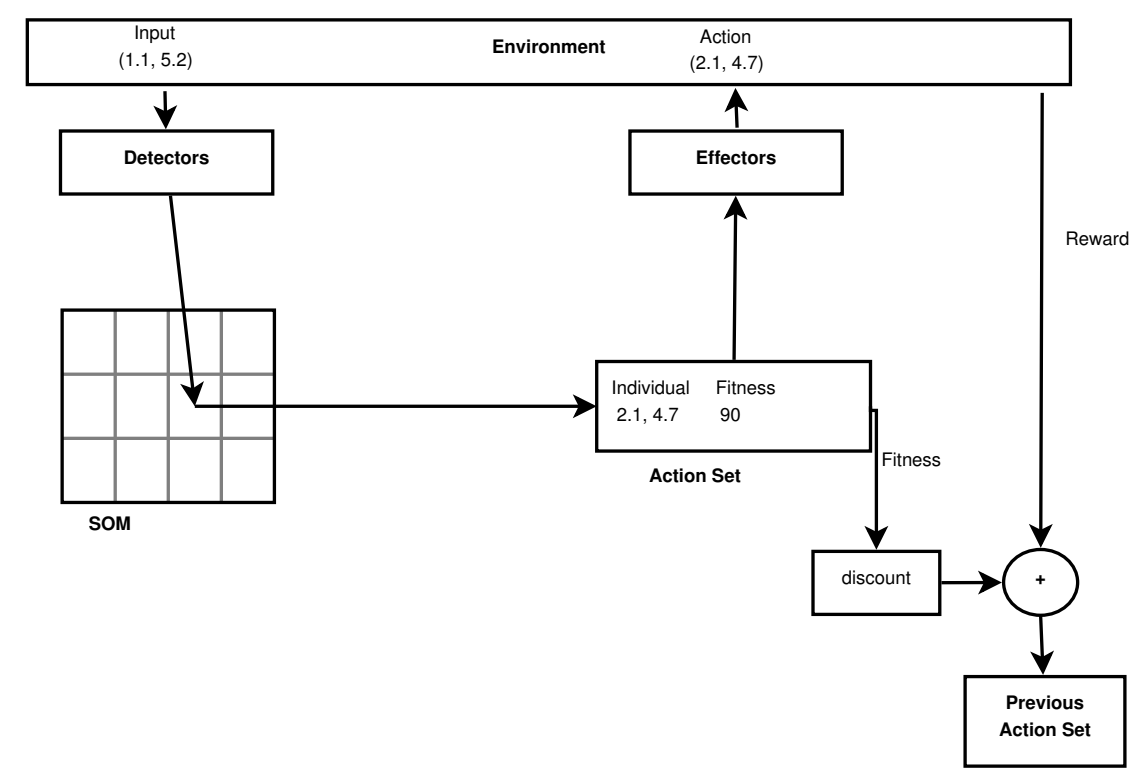

Figure 4: Self Organizing Classifier Schematic

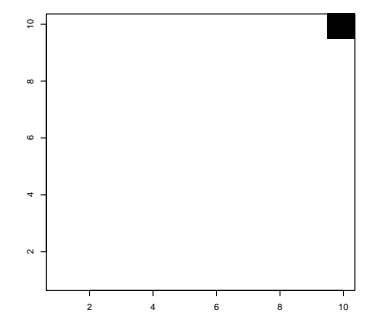

Figure 5: Empty room environment. The black position represents the goal.

depicted in Figures 5 and 6 . All environments require continuous actions. The variable observed by the agent is the agent's position which is also continuous.

At every trial, the agent starts at a random position on the environment. Naturally, starting inside a wall is not possible. Reaching the goal would give the agent a reward of 1000, hitting an obstacle would return -20 and any other action would return -10 . Additionally, agents can not move more than 1.0 in any direction. The collision system is simply implemented (which makes it harder than a real system). If an agent tries to move inside a wall, the system detects the infraction, sets the agent in the previous position and returns the reward. In other words, an agent constantly hitting the wall will not move at all. However, an agent that hits the limits of a maze will have its final position limited by the environment. Therefore, it is possible to move sideways when hitting the limit of the environment.

\subsection{Settings and Design of Experiments}

The parameters of the algorithm are fixed and described in Table 1. Here, it means the SOM iteration number, chebyshevDistance() is the Chebyshev distance between the current cell and the cell which won the SOM's competi-

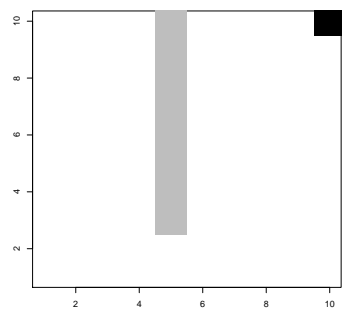

Figure 6: One-Wall Maze environment. Grey positions are obstacles and the black position is the goal.

tion and random $(a, b)$ is a function which returns a uniform random value between $a$ and $b$. The cells of the SOM are only updated if the neighborhood function multiplied by the learning restraint surpasses the cell update threshold.

Following the design of 20 the performance is computed as the average steps to reach the goal during the last 100 trials. The trials can not last more than 500 steps. Any trial which last more than 500 is terminated and a new trial is started with the agent, as usual, in a random position. All statistics, when not stated otherwise, are averaged over 20 experiments.

\subsection{Empty Room}

Empty Room is a well known problem, in continuous 14 as well as discrete action spaces 20. The evaluation focus here is on the overall functionality of the system. Although not complicated in nature, this problem may expose strong instabilities and other undesired phenomena.

Figure 7 shows the average behavior obtained. To construct this figure, the action of the agent was sampled 100 times and averaged in a given environment area of size $1 \mathrm{x} 1$. The resulting matrix have a dimension of 10x10 averaged ac- 
Table 1: Parameters

\begin{tabular}{|c|l|l|}
\hline Differential Evolution & CR & 0.2 \\
& F & random $(0,1)$ \\
\hline \multirow{5}{*}{ Self Organizing Map } & Matrix Size & $10 \times 10$ \\
& Weight's initial value & random $(0,1)$ \\
& Learning restraint & $0.1(0.999999)^{i t}$ \\
& Neighborhood function & exp $\left(-\right.$ chebyshevDistance ()$\left.^{2}\right)$ \\
& Cell update threshold & 0.005 \\
\hline \multirow{5}{*}{ Self Organizing Classifiers } & $\eta$ & 0.2 \\
& $\beta$ & 5 \\
& $\iota$ & 10 \\
& $\iota$ & 20 \\
& $\gamma$ & 0.9 \\
& InitialFitness & 0 \\
\hline
\end{tabular}

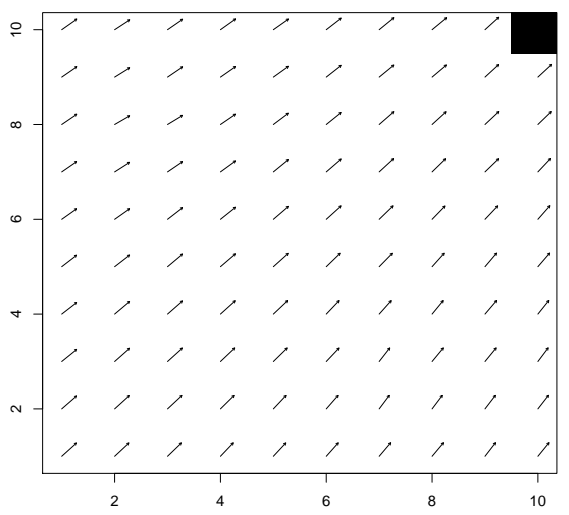

Figure 7: Behavior evolved in the Empty Room environment.

tions. Subsequently, the matrices of 20 experiments in the same environment were averaged.

The SOC's developed behavior is optimal, in spite of the fact that inside the SOM structure each cell must arrive at the best solution (fitness is not global). The justification lies in the fact that the ability of the SOC algorithm to share and improve solutions between cells make the development very fast and stable. Figure 8 shows the performance. The fitness distribution is calculated in the same way as the matrix of actions, but instead of action vectors the maximum fitness of the cell is measured. The result is shown on Figure 9.

\subsection{One-Wall Maze}

This second problem is different from the previous one. It makes necessary the use of different actions for different places of the environment. Note that the classifiers used are very simple. Therefore, this experiment evaluates specifically the dynamical population structure's niching capabilities.

Figure 10 shows the behavior of the agent in the OneWall Maze environment. This figure was obtained in the same manner of Figure 7

One-Wall Maze is a difficult problem. Even so, the behavior observed is accurate and stable. This is justifiable by the SOM population capability of first self-organizing to the

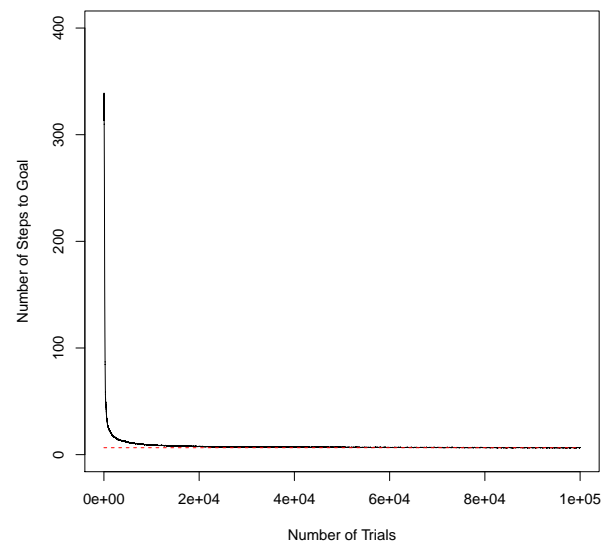

Figure 8: Performance over the Empty Room environment (continuous line). For guiding purposes a dashed line is drawn at 6.5 of distance to the $\mathrm{x}$ coordinate.

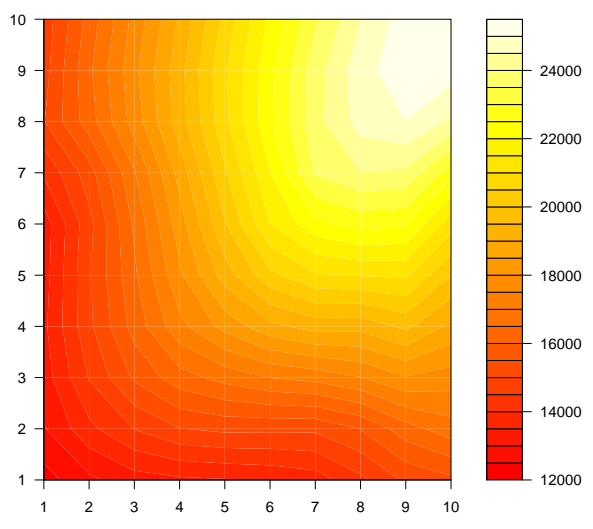

Figure 9: Average distribution of fitness in the Empty Room environment. 


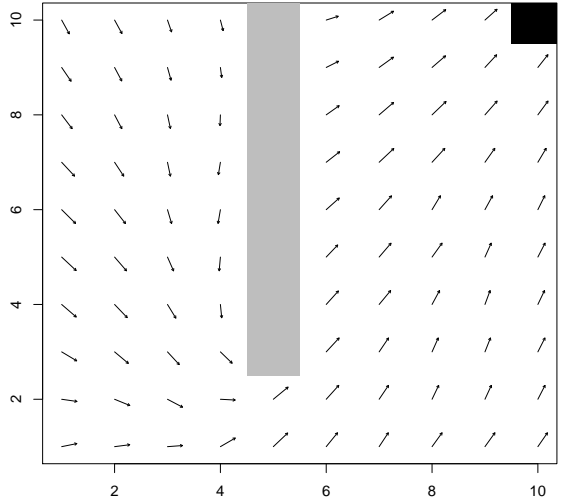

Figure 10: Behavior evolved in the One-Wall Maze environment.

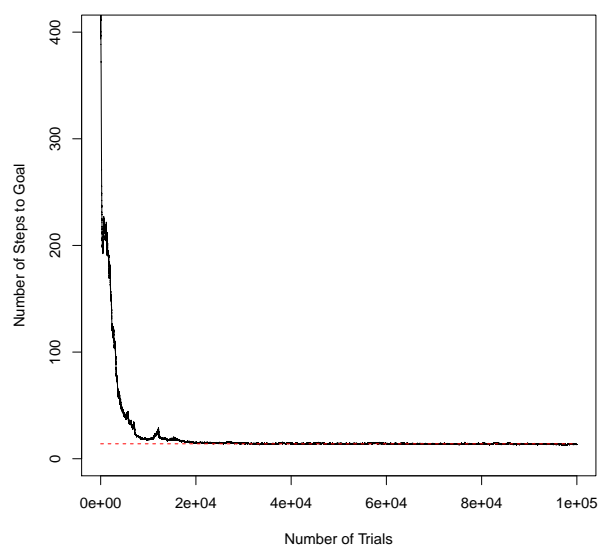

Figure 11: Performance over the One-Wall Maze environment (continuous line). Dashed-line is drawn for guiding purposes parallel to the $\mathrm{x}$-coordinate at 14 of distance.

environment's input distribution. Projecting and dividing the input space respecting its topology. And secondly, the ability of developing different behavior at different parts of the space (niches) with their own subpopulation.

Note that the SOM used was initialized to values between zero and one as weight vectors. Therefore, it was not in any way biased to the environment's inputs range (environment values range from zero to ten). Additionally, Figures 11 and 12 show respectively the performance and fitness distribution.

\subsection{Population Analysis}

But what is the cost of running a SOC? Solving a problem is often not enough, it is required that a method uses reasonably its resources. The aim of this section is to evaluate briefly the population requirements of SOC. All tests are run over the One-Wall Maze.

Foremost, SOC's maximum possible population size can

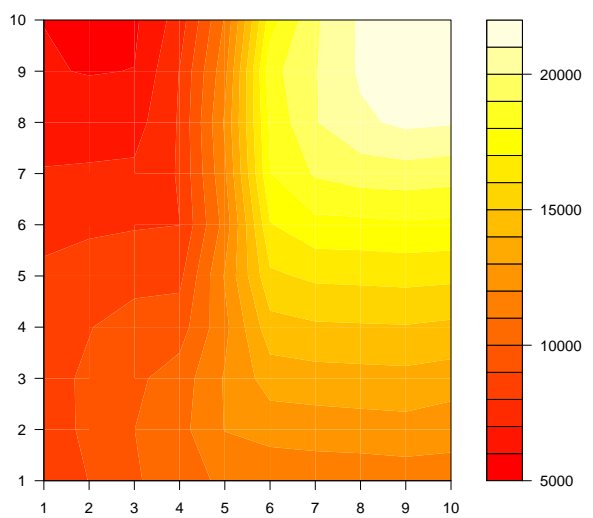

Figure 12: Average distribution of fitness in the One-Wall Maze environment.

be computed by the following equation:

$$
\text { MaximumPopulationSize }=\text { cells } *(\beta+\nu),
$$

where cells is the number of cells present in the SOM map. Taking the parameters found in Table 11 it is possible to compute a maximum population size of 1500 for the previous experiments. However, as shown in Figure 13 this value is never reached. The population grows rapidly to around 1100 and remains stable below this value.

Moreover, Figure 13 shows the population growth of two other variations of the same algorithm. Test $\mathrm{A}$ has $\beta=2$ and $\nu=5$ resulting in a maximum population size of 700 , while Test $\mathrm{B}$ has a $7 \times 7$ SOM map and therefore having 735 of maximum population size. Note that they reach around 500 individuals and after that the population size remains approximately constant.

Figures 14 and 15 show respectively the behavior of Test $\mathrm{A}$ and Test B. Both achieve similar actions to the original algorithm with a bigger population. Although, Test A have places with high direction variations which result in vectors with smaller magnitude. This happens because smaller population sizes enable frequently accessed cells to evolve faster which makes even more infrequent the access of spots near the wall. Consequently, the SOM may not have any cells mapping those infrequent spots near the wall.

Tests A and B performance is also similar (see Figure 16. Actually, Tests A and B achieves the optimum faster than the original, because their populations are smaller, however, Test B presents small deviations from the optimum.

Thus, the cost in terms of population size is small. Besides, as shown above, smaller populations have similar results. Recall that a neural based LCS needed a maximum population size of 16000 individuals to solve a continuous Empty Room problem 14. And XCS required on average 404 macroclassifiers to solve a similar problem though with discrete output called Side 10 20]. Therefore, SOC's results are promising when compared with the literature.

\section{CONCLUSIONS}

This paper proposed the algorithm named Self Organizing 


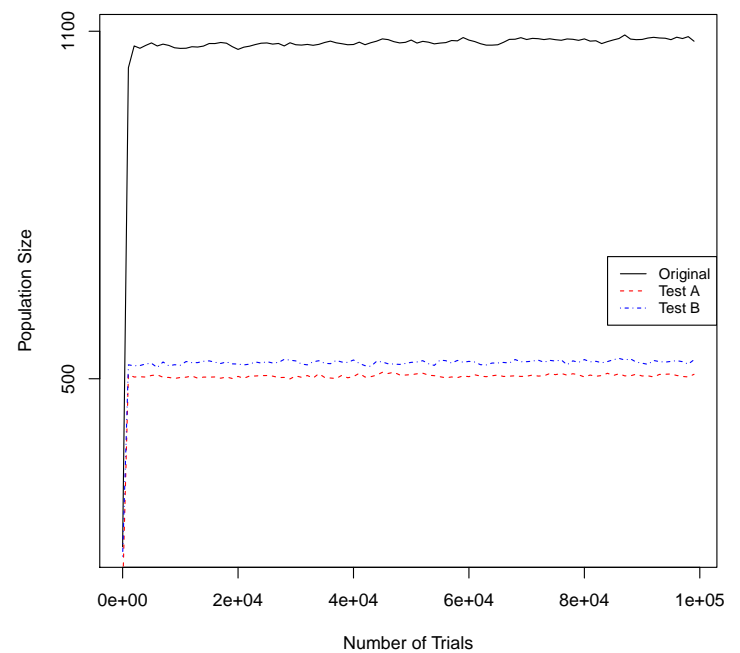

Figure 13: Population dynamics for the original and two additional tests with smaller maximum populations.

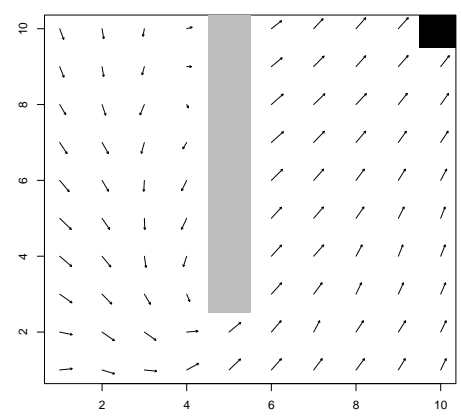

Figure 14: Behavior for the algorithm with a smaller population, i.e., $\beta=2$ and $\nu=5$ (Test A).

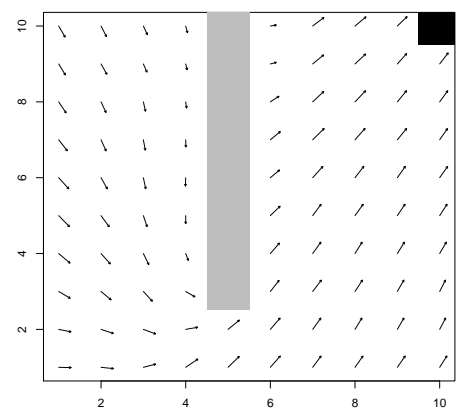

Figure 15: Behavior for the algorithm with a smaller population, i.e., a $7 \times 7 \mathrm{SOM}$ population (Test $\mathrm{B})$.

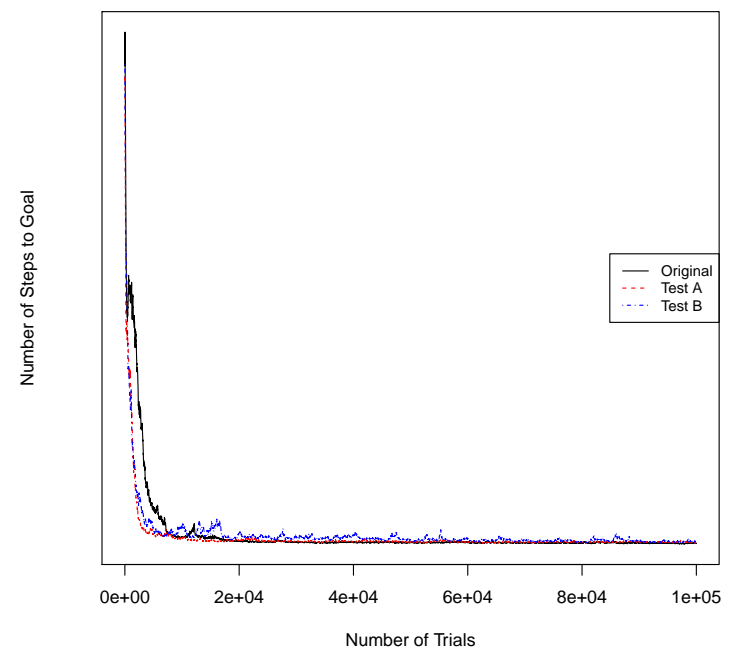

Figure 16: Performance comparison between the original parameters and the smaller population versions (Tests A and B).

Classifiers which possess a different perspective on how to face the balance of generalized and specialized classifiers.

We showed that with a self-organizing structured population, the separation of niching and fitness pressures is possible. Experiments on continuous multi-step problems showcased the capabilities of the approach. Note that this article presented a problem which is more difficult than previous continuous multi-step problems. This is just the first article about this type of algorithm and the difficulty of the problems solved shows a promising horizon for this line of algorithms.

Additionally, two new concepts were introduced:

- Niched Fitness - a fitness relative to the niche;

- SOM Population - a dynamical structured population based on the SOM's dynamics.

Both concepts are fundamental to understand the SOC algorithm.

Thus, with the promising results accomplished by the novel concepts and the algorithm introduced here, they will, hopefully, aid the development of other algorithms and theories specially in the area of genetics based machine learning or evolutionary computation in general.

\section{REFERENCES}

[1] E. Alba and M. Tomassini. Parallelism and evolutionary algorithms. Evolutionary Computation, IEEE Transactions on, 6(5):443-462, 2002.

[2] E. Alba and J. Troya. Cellular evolutionary algorithms: Evaluating the influence of ratio. In Parallel Problem Solving from Nature PPSN VI, pages 29-38. Springer, 2000.

[3] T. Belding. The distributed genetic algorithm revisited. In Proceedings of the Sixth International Conference on Genetic Algorithms: University of 
Pittsburgh, July 15-19, 1995, page 114. Morgan Kaufmann Pub, 1995.

[4] A. Bonarini, C. Bonacina, and M. Matteucci. Fuzzy and crisp representations of real-valued input for learning classifier systems. Learning Classifier Systems, pages 107-124, 2000.

[5] L. Bull. On using constructivism in neural classifier systems. Parallel problem solving from nature-PPSN VII, pages 558-567, 2002.

[6] L. Bull and T. O'Hara. Accuracy-based neuro and neuro-fuzzy classifier systems. In Proceedings of the Genetic and Evolutionary Computation Conference, pages 905-911. Morgan Kaufmann Publishers Inc., 2002.

[7] M. Butz and O. Herbort. Context-dependent predictions and cognitive arm control with XCSF. In Proceedings of the 10th annual conference on Genetic and evolutionary computation, pages 1357-1364. ACM, 2008.

[8] M. Butz, T. Kovacs, P. Lanzi, and S. Wilson. Toward a theory of generalization and learning in XCS. Evolutionary Computation, IEEE Transactions on, 8(1):28-46, 2004.

[9] M. Butz, P. Lanzi, and S. Wilson. Function approximation with XCS: Hyperellipsoidal conditions, recursive least squares, and compaction. Evolutionary Computation, IEEE Transactions on, 12(3):355-376, 2008.

[10] M. Butz and S. Wilson. An algorithmic description of XCS. Advances in Learning Classifier Systems, pages 267-274, 2001.

[11] J. Casillas, B. Carse, and L. Bull. Fuzzy-XCS: A michigan genetic fuzzy system. Fuzzy Systems, IEEE Transactions on, 15(4):536-550, 2007.

[12] S. García, D. Molina, M. Lozano, and F. Herrera. A study on the use of non-parametric tests for analyzing the evolutionary algorithms' behaviour: a case study on the CEC'2005 special session on real parameter optimization. Journal of Heuristics, 15(6):617-644, 2009.

[13] J. Horn, D. Goldberg, and K. Deb. Implicit niching in a learning classifier system: Nature's way. Evolutionary Computation, 2(1):37-66, 1994.

[14] G. Howard, L. Bull, and P. Lanzi. Towards continuous actions in continuous space and time using self-adaptive constructivism in neural XCSF. In Proceedings of the 11th Annual conference on Genetic and evolutionary computation, pages 1219-1226. ACM, 2009.

[15] G. Hutchinson. Concluding remarks. In Cold Spring Harbor Symposia on Quantitative Biology 22, pages 415-427, 1957.

[16] A. Iorio and X. Li. Solving rotated multi-objective optimization problems using differential evolution. AI 2004: Advances in Artificial Intelligence, pages 861-872, 2005.

[17] M. Iqbal, W. N. Browne, and M. Zhang. Xcsr with computed continuous action. In AI 2012: Advances in Artificial Intelligence, pages 350-361. Springer, 2012.

[18] T. Kohonen. The self-organizing map. Proceedings of the IEEE, 78(9):1464-1480, 1990.
[19] P. Lanzi et al. A study of the generalization capabilities of XCS. In Proceedings of the Seventh International Conference on Genetic Algorithm, pages 418-425, 1997.

[20] P. Lanzi, D. Loiacono, S. Wilson, and D. Goldberg. XCS with computed prediction in multistep environments. In Proceedings of the 2005 conference on Genetic and evolutionary computation, pages 1859-1866. ACM, 2005.

[21] P. Lanzi, D. Loiacono, S. Wilson, and D. Goldberg. Generalization in the XCSF classifier system: Analysis, improvement, and extension. Evolutionary Computation, 15(2):133-168, 2007.

[22] P. Lanzi and R. Riolo. A roadmap to the last decade of learning classifier system research (from 1989 to 1999). Learning Classifier Systems, pages 33-61, 2000.

[23] B. Manderick and P. Spiessens. Fine-grained parallel genetic algorithms. In ICGA'89, pages 428-433, 1989.

[24] J. Mouret. Novelty-based multiobjectivization. New Horizons in Evolutionary Robotics, pages 139-154, 2011.

[25] P. Stalph and M. Butz. Learning local linear jacobians for flexible and adaptive robot arm control. Genetic programming and evolvable machines, 13(2):137-157, 2012.

[26] R. Storn and K. Price. Differential evolution-a simple and efficient heuristic for global optimization over continuous spaces. Journal of global optimization, 11(4):341-359, 1997.

[27] M. Tomassini. Spatially structured evolutionary algorithms. Springer, 2005.

[28] H. Tran, C. Sanza, Y. Duthen, and T. Nguyen. XCSF with computed continuous action. In Genetic And Evolutionary Computation Conference: Proceedings of the 9 th annual conference on Genetic and evolutionary computation, volume 7, pages 1861-1869, 2007.

[29] R. Urbanowicz and J. Moore. Learning classifier systems: a complete introduction, review, and roadmap. Journal of Artificial Evolution and Applications, 2009:1, 2009.

[30] M. Valenzuela-Rendón. The fuzzy classifier system: A classifier system for continuously varying variables. In Proceedings of the Fourth International Conference on Genetic Algorithms pp346-353, Morgan Kaufmann I, volume 991, pages 223-230, 1991.

[31] J. Vesterstrom and R. Thomsen. A comparative study of differential evolution, particle swarm optimization, and evolutionary algorithms on numerical benchmark problems. In Evolutionary Computation, 2004. CEC2004. Congress on, volume 2, pages 1980-1987. IEEE, 2004.

[32] B. Widrow and M. E. Hoff. Adaptive Switching Circuits. In 1960 IRE WESCON Convention Record, Part 4, pages 96-104, New York, 1960. IRE.

[33] S. Wilson. ZCS: A zeroth level classifier system. Evolutionary computation, 2(1):1-18, 1994.

[34] S. Wilson. Classifier fitness based on accuracy. Evolutionary computation, 3(2):149-175, 1995.

[35] S. W. Wilson. Classifiers that approximate functions. 1(2-3):211-234, June 2002. 